\title{
The size of the maximum antichains in products of linear orders
}

\author{
Denis Bouyssou • Thierry Marchant • \\ Marc Pirlot
}

Received: date / Accepted: date

\begin{abstract}
The size of maximum antichains in the product of $n$ linear orders is known when the $n$ linear orders have the same length. We present an exact expression for the size of maximum antichains when the linear orders have (possibly) different lengths. From this, we derive an exact expression for the size of maximum antichains in the product of $n$ linear orders with the same length. This expression is equivalent to but different from the existing expression. It allows us to present an asymptotic result for the size of maximum antichains of $n$ linear orders with the same length $m$ going to infinity.
\end{abstract}

Keywords maximal antichain - Sperner - multichoice cooperative game . linear orders

Mathematics Subject Classification (2010) $91 \mathrm{~B} 06$

\section{Introduction}

Antichains in the poset $\{0,1\}^{n}$ equipped with the standard partial ordering are well-studied and have many different interpretations [4]. An expression for the maximal size of such antichains in $\{0,1\}^{n}$ is given by a classical theorem due to

Authors are listed alphabetically and have equally contributed.

D. Bouyssou (Orcid: 0000-0003-3487-8498)

LAMSADE, UMR 7243, CNRS, Université Paris-Dauphine, PSL Research University, 75016

Paris, France

E-mail: bouyssou@lamsade.dauphine.fr

T. Marchant (Orcid: 0000-0002-2893-2964)

Ghent University, Dunantlaan 1, 9000 Ghent, Belgium

E-mail: thierry.marchant@ugent.be

M. Pirlot (Orcid: 0000-0002-3689-0944)

Université de Mons, rue de Houdain 9, 7000 Mons, Belgium

E-mail: marc.pirlot@umons.ac.be 
Sperner [19]. If we consider the more general poset $\{1, \ldots, m\}^{n}$ also equipped with the standard partial ordering, an expression for the size of maximum antichains is given by Sander [17]. Sander also provides asymptotic results when $m$ is fixed and $n$ goes to infinity. The interest of Sander in this problem arose from a recreational mathematics problem posed in [14]. Actually, antichains and, hence, maximal antichains, in the poset $\{1, \ldots, m\}^{n}$ are of interest in many domains. For instance in game theory, [10] define a multichoice cooperative game as a real-valued mapping on $\{1, \ldots, m\}^{n}$, where $n$ is the number of players and $\{1, \ldots, m\}$ denotes the set of ordered actions that each player can take. A profile in such a game is a vector $x=\left(x_{1}, \ldots, x_{n}\right) \in\{1, \ldots, m\}^{n}$ and represents the actions taken by each agent. A winning profile is such that the value of the game at that profile is 1 . A winning profile $x$ is minimal if there is no other winning profile $y$ such that $y \leq x$. If a game is monotone, then the set of all minimal winning profiles is an antichain. Besides, [7] shows that antichains in $\{1, \ldots, m\}^{n}$ play an important role in the analysis of these multichoice cooperative games.

In [9], a generalization of multichoice cooperative games is presented by considering that the set of actions available to agent $i$ is $\left\{1, \ldots, m_{i}\right\}$. A multichoice cooperative game thus becomes a real-valued mapping on $\prod_{i=1}^{n}\left\{1, \ldots, m_{i}\right\}$. Applications of multichoice cooperative games in various domains (cost allocation, voting, ... ) can be found in $[2,6]$. A very simple example ${ }^{1}$ of multi-choice cooperative game is as follows. Consider a voting situation in a parliament in which the players are the political parties, $m_{i}$ represents the number of seats of party $i$, a winning profile is such that the proposal obtains enough votes to be approved, and there is no voting discipline in political parties. In this setting, minimal winning profiles are antichains and they are interesting because they give information about how to approve a proposal.

Our personal interest in antichains in the poset $\prod_{i=1}^{n}\left\{1, \ldots, m_{i}\right\}$ stems from the analysis of a multicriteria sorting model (called ELECTRE TRI-nB) presented in [5]. This model, when restricted to two categories, makes use of $p$ limiting profiles (vectors of length $n$ ) supposed to lie at the border between the upper and the lower category. An alternative $x$ (also a vector of length $n$ ) is assigned to the upper category if $x$ is weakly preferred to at least one limiting profile and no limiting profile is strictly preferred to $x$. In this context, the size of maximal antichains corresponds to the maximum number of limiting profiles needed to represent a twofold ordered partition in ELECTRE TRI-nB, whenever such a representation is possible. See [1] for a detailed explanation of the relation between antichains and ELECTRE TRI-nB.

Another paper about antichains in $\{1, \ldots, m\}^{n}$ is [20] (and the references therein): it presents an upper bound for the number of antichains (a generalization of Dedekind numbers).

In the present paper, we extend Sander's results in two directions. First, we present an exact expression for the size of maximum antichains in the heterogeneous product $\prod_{i=1}^{n}\left\{1, \ldots, m_{i}\right\}$. Then, we provide asymptotic results

1 This example was suggested to us by an anonymous referee. 
for the size of maximum antichains in $\{1, \ldots, m\}^{n}$ when $n$ is fixed and $m$ goes to infinity.

The rest of the paper is organized as follows. In Section 2, we introduce some notation and the main definitions. Section 3 is devoted to the general case of heterogeneous products and presents some exact results about the size of maximum antichains. Our main results are presented in Section 4, in which we consider the special case of homogeneous products and we present a new exact result and also an asymptotic one when $n$ is fixed.

\section{Notation and definitions}

Let $P$ be a set and $\leq$ be a binary relation defined on $P$, satisfying (i) reflexivity $(\forall x \in P, x \leq x)$, (ii) antisymmetry $(\forall x, y \in P, x \leq y$ and $y \leq x \Longleftrightarrow x=y)$ and (iii) transitivity $(\forall x, y, z \in P, x \leq y$ and $y \leq z \Rightarrow x \leq z)$. The pair $(P, \leq)$ is called a partially ordered set $(\text { poset })^{2}$. When there is no ambiguity, the poset $(P, \leq)$ is simply denoted by $P$. For all $x, y$ belonging to a poset $P$, we say that $x$ and $y$ are comparable if $x \leq y$ or $y \leq x$. A chain of $P$ is a totally ordered subset of $P$. A linear order on $P$ is a poset such that $P$ is a chain. An antichain of $P$ is a subset of pairwise incomparable elements. A maximum antichain is an antichain of maximal cardinality.

Let $\left(P, \leq_{P}\right)$ and $\left(Q, \leq_{Q}\right)$ be two posets. The product poset $(P \times Q, \leq)$ is defined to be the set of all pairs $(a, b), a \in P, b \in Q$, with the order given by $(a, b) \leq\left(a^{\prime}, b^{\prime}\right)$ if and only if $\left(a \leq_{P} a^{\prime}\right)$ and $\left(b \leq_{Q} b^{\prime}\right)$. Let $n$ be a positive integer and $\bar{m}=\left(m_{1}, \ldots, m_{n}\right)$ be an element of $\mathbb{N}^{n}$, where $\mathbb{N}$ denotes the set of positive integers. For any $a \in \mathbb{N}$, let $[a]$ denote the set $\{1, \ldots, a\}$. For any $i \in[n]$, the poset $\left(\left[m_{i}\right], \leq\right)$, where $\leq$ is the usual ordering of the natural numbers, is a linear order (also called a chain). The product of these $n$ chains is the poset $\left(\prod_{i=1}^{n}\left[m_{i}\right], \preceq\right)$ where $\preceq$ is defined as follows: for all $x, y \in \prod_{i=1}^{n}\left[m_{i}\right]$, $x \preceq y$ iff $x_{i} \leq y_{i}$ for all $i \in[n]$ [8]. When $\bar{m}$ is such that $m_{i}=m$ for all $i \in[n]$, then the Cartesian product $\prod_{i=1}^{n}\left[m_{i}\right]$ is homogeneous and can be written as $[m]^{n}$.

The size of the maximum antichains in $\prod_{i=1}^{n}\left[m_{i}\right]$ and $[m]^{n}$ is respectively denoted by $s(\bar{m})$ and $S(m, n)$. [19] has proved that the size of a maximum antichain in $[2]^{n}$ is

$$
S(2, n)=\left(\begin{array}{c}
n \\
\lfloor n / 2\rfloor
\end{array}\right)
$$

When $n$ is large, a convenient approximation for $S(2, n)$ is obtained using Stirling's formula: $S(2, n) \sim 2^{n} \sqrt{2 / \pi n}$. Later, [17] has proved that the size of a maximum antichain in $[\mathrm{m}]^{n}$ is

$$
S(m, n)=\sum_{j=0}^{\lfloor g / m\rfloor}(-1)^{j}\left(\begin{array}{l}
n \\
j
\end{array}\right)\left(\begin{array}{c}
n-1+g-m j \\
n-1
\end{array}\right),
$$

\footnotetext{
2 Most definitions about posets are taken from [16].
} 
with $g=\lfloor n(m-1) / 2\rfloor$. Sander has also provided a bound ${ }^{3}$ and some asymptotic results for $S(m, n)$ when $m$ is fixed. Asymptotic results for $S(m, n)$ when $n$ is fixed have not been discussed in the literature. Notice that $S(m, n)$ corresponds to Sequence A077042 in the On-line Encyclopedia of Integer Sequences $[15]$.

\section{Heterogeneous product}

Since multichoice cooperative games have been generalized to heterogeneous sets of actions [9] and the analysis of ELECTRE TRI-nB also involves antichains in a heterogeneous product set [1], we need results about $s(\bar{m})$. Let us define $m_{I}=\sum_{i \in I} m_{i}$ and

$$
h=\left\lfloor\frac{n+m_{[n]}}{2}\right\rfloor .
$$

An expression for $s(\bar{m})$ was posted on February 11, 2013, by Brian M. Scott on the website StatExchange [18]. ${ }^{4}$

Theorem 1 For all $\bar{m}=\left(m_{1}, \ldots, m_{n}\right) \in \mathbb{N}^{n}$,

$$
s(\bar{m})=\sum_{I \subseteq[n]: m_{I} \leq h-n}\left(\begin{array}{c}
h-m_{I}-1 \\
n-1
\end{array}\right)(-1)^{|I|} .
$$

Since the website only presents an informal and elliptic proof, we deem it useful to present a formal proof below. Let us first recall some definitions and results about posets. Let $(P, \leq)$ be a poset. For any $x, y \in P$, we say that $y$ covers $x$ in $P$ iff $x<y$ and there is no $z$ such that $x<z<y$. A ranking (or grading) of a poset $P$ is a partition of $P$ into (possibly empty) sets $P_{i}(i \in \mathbb{Z})$ such that, for each $i$, every element in $P_{i}$ is covered only by elements in $P_{i+1}$. The set $P_{i}$ is called the $i$ th rank of $P$. If a poset admits a ranking, then we say that it is ranked (or graded). $P$ is said to be Sperner if every rank of largest size is a maximum antichain.

Proof of Theorem 1. In this proof, for the sake of brevity, we use $X$ to denote the poset $\left(\prod_{i=1}^{n}\left[m_{i}\right], \preceq\right)$ or the set $\prod_{i=1}^{n}\left[m_{i}\right]$. For each $i \in[n]$, the poset $\left(\left[m_{i}\right], \leq\right)$ is a chain. Hence, $X$ is a product of chains, it is Sperner and the median rank (or ranks if $n+m_{[n]}$ is odd) is a maximum antichain of $X[3$, 8]. It is simple to see that this rank is the set

$$
D=\left\{x \in X: \sum_{i \in[n]} x_{i}=h\right\} .
$$

\footnotetext{
3 This bound is later rediscovered by [12] in a different context. In addition, [12] note that (1) can be found in [13] as the solution of a probability problem.

4 When we submitted the first version of this paper, we were not aware of Scott's post and our proof used generating functions. We thank an anonymous reviewer for pointing to us this post on StatExchange.
} 
For any $I \subseteq[n]$, let us define

$$
D_{I}=\left\{x \in \mathbb{N}^{n}: \sum_{i \in[n]} x_{i}=h \text { and } x_{i}>m_{i} \forall i \in I\right\} .
$$

We have $D_{I}=\bigcap_{i \in I} D_{\{i\}}$ and using the multiset coefficient formula (also known as 'stars and bars' technique), we find

$$
\left|D_{I}\right|=\left(\begin{array}{c}
h-1-m_{I} \\
n-1
\end{array}\right) \text {. }
$$

It is clear that

$$
D=D_{\emptyset} \backslash \bigcup_{i \in[n]} D_{\{i\}} .
$$

Using the inclusion-exclusion principle, we find

$$
\begin{aligned}
|D|=\left|D_{\emptyset}\right|-\left|\bigcup_{i \in[n]} D_{\{i\}}\right| & =\left|D_{\emptyset}\right|-\sum_{\emptyset \neq I \subseteq[n]}(-1)^{|I|+1}\left|\bigcap_{i \in I} D_{\{i\}}\right| \\
& =\left|D_{\emptyset}\right|+\sum_{\emptyset \neq I \subseteq[n]}(-1)^{|I|}\left|D_{I}\right| \\
& =\sum_{I \subseteq[n]}(-1)^{|I|}\left|D_{I}\right| \\
& =\sum_{I \subseteq[n]}(-1)^{|I|}\left(\begin{array}{c}
h-m_{I}-1 \\
n-1
\end{array}\right) .
\end{aligned}
$$

Since $\left(\begin{array}{c}h-m_{I}-1 \\ n-1\end{array}\right)=0$ whenever $h-m_{I}<n$, we obtain (2).

Table 1 illustrates how $s(\bar{m})$ varies as a function of $\bar{m}$. In particular, we

\begin{tabular}{cr}
\hline$m_{1}, \ldots, m_{n}$ & size \\
\hline 5,5 & 5 \\
$5,5,5$ & 19 \\
$5,5,10$ & 25 \\
$5,5,100$ & 25 \\
$5,5,10,10$ & 210 \\
$5,5,10,20$ & 250 \\
$5,5,10,100$ & 250 \\
$10,10,10,10$ & 670 \\
$10,10,10,20$ & 1000 \\
$10,10,10,100$ & 1000 \\
$100,100,100,100$ & 666700 \\
$10, \ldots, 10(10$ times $)$ & 432457640 \\
$100, \ldots, 100(10$ times $)$ & 430438025018583040 \\
\hline
\end{tabular}

Table 1 The size of the maximum antichains with $m_{1}, \ldots, m_{n}$ levels on $n$ attributes.

see that increasing one of the $m_{i}$ 's way above the others has a limited impact. 
When all components of $\bar{m}$ are identical, it is easy to show that (2) coincides with Sander's expression (1). In that case, Sander's expression is computationally more efficient than ours.

This section about heterogeneous products does not contain any asymptotic result because it does not seem relevant to let one of the parameters, say $m_{5}$, go to infinity while keeping the other parameters constant.

\section{Homogeneous product}

In addition to an exact expression for $S(m, n)$, [17] provides asymptotic results when $n \rightarrow \infty$. Our goal in this section is to analyze the asymptotic behavior of $S(m, n)$ when $m \rightarrow \infty$. This corresponds in multichoice simple games to the case in which the number of ordered actions the players can take is very large. In the case of multicriteria sorting, this corresponds to situations in which the alternatives to be sorted are evaluated on criteria scales involving a large number of levels. To this end, we first present a new exact expression for $S(m, n)$ from which we then derive an asymptotic result.

Let $h=\lfloor n(m+1) / 2\rfloor$. Our first result about homogeneous products is the following.

Theorem 2 For all $n \geq 2$, if $n(m+1)$ is even, then $S(m, n)$ is equal to

$$
m^{n-1} \quad-\quad 2 \sum_{r=n-1}^{h-m-1} \quad \sum_{i=0}^{\left\lfloor\frac{r-n+1}{m-1}\right\rfloor}(-1)^{i}\left(\begin{array}{c}
n-1 \\
i
\end{array}\right)\left(\begin{array}{c}
r-i m-1 \\
r-i m-n+1
\end{array}\right)
$$

Otherwise, $S(m, n)$ is equal to

$$
\begin{aligned}
m^{n-1}-2 \sum_{r=n-1}^{h-m-1} \sum_{i=0}^{\left\lfloor\frac{r-n+1}{m-1}\right\rfloor} & (-1)^{i}\left(\begin{array}{c}
n-1 \\
i
\end{array}\right)\left(\begin{array}{c}
r-i m-1 \\
r-i m-n+1
\end{array}\right) \\
& -\sum_{i=0}^{\left\lfloor\frac{h-m-n+1}{m-1}\right\rfloor}(-1)^{i}\left(\begin{array}{c}
n-1 \\
i
\end{array}\right)\left(\begin{array}{c}
h-i m-1 \\
h-i m-n+1
\end{array}\right) .
\end{aligned}
$$

Proof. We first prove (3). We have seen in the proof of Theorem 1 that an antichain of maximum size in $\prod_{i \in[n]}\left[m_{i}\right]$ is the set $A=\left\{x \in X: \sum_{i \in[n]} x_{i}=\right.$ $h\}$ where $h=\left\lfloor\frac{n+\sum_{i \in[n]} m_{i}}{2}\right\rfloor$. Hence, if $n(m+1)$ is even, then an antichain of maximum size in $[m]^{n}$ is the set $A=\left\{x \in[m]^{n}: \sum_{i \in[n]} x_{i}=h\right\}$, with $h=n(m+1) / 2$. Since $1 \leq x_{n} \leq m$, if we project the set $A$ on $[m]^{n-1}$ by dropping the last coordinate $x_{n}$, we obtain the set $A^{\prime}=\left\{y \in[m]^{n-1}\right.$ : $\left.h-m \leq \sum_{i \in[n-1]} y_{i} \leq h-1\right\}$. Since no $x, y \in A$ are comparable, we know that no distinct $x, y \in A$ project on the same element in $[m]^{n-1}$. Hence $\left|A^{\prime}\right|=|A|$ 
and $S(m, n)$ is equal to

$$
\begin{aligned}
\left|A^{\prime}\right|= & m^{n-1}-\left|\left\{y \in[m]^{n-1}: \sum_{i \in[n-1]} y_{i} \leq h-m-1\right\}\right| \\
& -\left|\left\{y \in[m]^{n-1}: \sum_{i \in[n-1]} y_{i} \geq h\right\}\right| \\
= & m^{n-1}-2\left|\left\{y \in[m]^{n-1}: \sum_{i \in[n-1]} y_{i} \leq h-m-1\right\}\right|,
\end{aligned}
$$

where the last equality holds because

$$
h-m-1-\min _{y \in[m]^{n-1}} \sum_{i \in[n-1]} y_{i}=\max _{y \in[m]^{n-1}} \sum_{i \in[n-1]} y_{i}-h .
$$

Let us rewrite $\left\{y \in[m]^{n-1}: \sum_{i \in[n-1]} y_{i} \leq h-m-1\right\}$ as the union of several sets:

$$
\bigcup_{r=n-1}^{h-m-1} A_{r} \text { where } A_{r}=\left\{y \in[m]^{n-1}: \sum_{i \in[n-1]} y_{i}=r\right\} .
$$

Clearly, for any $r \neq s, A_{r} \cap A_{s}=\emptyset$ and

$$
S(m, n)=m^{n-1}-2 \sum_{r=n-1}^{h-m-1}\left|A_{r}\right| .
$$

Let

$$
\begin{aligned}
& B_{r}=\left\{y \in \mathbb{N}^{n-1}: \sum_{i \in[n-1]} y_{i}=r\right\} \\
& C_{r}^{l}=\left\{y \in \mathbb{N}^{n-1}: \sum_{i \in[n-1]} y_{i}=r \text { and } y_{l}>m\right\} \text { for } l \in[n-1]
\end{aligned}
$$

and $C_{r}^{l *}=B_{r} \backslash C_{r}^{l}$. Then $A_{r}=\bigcap_{l \in[n-1]} C_{r}^{l *}$ and, thanks to the inclusionexclusion principle,

$$
\begin{aligned}
& \left|A_{r}\right|=\left|B_{r}\right|-\left|\bigcup_{l \in[n-1]} C_{r}^{l}\right| \\
& =\left|B_{r}\right|-\sum_{\emptyset \neq J \subseteq[n-1]}(-1)^{|J|-1}\left|\bigcap_{l \in J} C_{r}^{l}\right| \\
& =\left|B_{r}\right|-\sum_{[i]: 1 \leq i<n}(-1)^{i-1}\left(\begin{array}{c}
n-1 \\
i
\end{array}\right)\left|\bigcap_{l \in[i]} C_{r}^{l}\right| \text {, }
\end{aligned}
$$


where the last equality holds because all dimensions play the same role. The set $B_{r}$ is a regular $(n-2)$-dimensional simplex. Its cardinality is equal to the $(r-n+1)$-th simplicial polytope number in $n-2$ dimensions [11], that is

$$
\left|B_{r}\right|=\left(\begin{array}{c}
r-1 \\
r-n+1
\end{array}\right)
$$

The set $\bigcap_{l \in[i]} C_{r}^{l}$ is the set of all elements of $\mathbb{N}^{n-1}$ such that at least $i$ components are strictly larger than $m$. If $r<i(m-1)+n-1$, then $\bigcap_{l \in[i]} C_{r}^{l}$ is empty because it is not possible to have at least $i$ components strictly larger than $m$. Hence

$$
\left|A_{r}\right|=\left|B_{r}\right|-\sum_{[i]: 1 \leq i \leq j}(-1)^{i-1}\left(\begin{array}{c}
n-1 \\
i
\end{array}\right)\left|\bigcap_{l \in[i]} C_{r}^{l}\right|
$$

where $j$ is the largest integer such that $r \geq j(m-1)+n-1$. If $r \geq i(m-1)+n-1$, then

$$
\left|\bigcap_{l \in[i]} C_{r}^{l}\right|=\left|B_{r-i m}\right|=\left(\begin{array}{c}
r-i m-1 \\
r-i m-n+1
\end{array}\right) .
$$

Combining (5), (6), (7) and (8) concludes the proof of (3).

The proof of (4) is similar. The main difference is that

$$
\begin{aligned}
\left|A^{\prime}\right|=m^{n-1}-2 & \left|\left\{y \in[m]^{n-1}: \sum_{i \in[n-1]} y_{i} \leq h-m-1\right\}\right| \\
& -\left|\left\{y \in[m]^{n-1}: \sum_{i \in[n-1]} y_{i}=h-m\right\}\right|,
\end{aligned}
$$

because

$$
h-m-1-\min _{y \in[m]^{n-1}} \sum_{i \in[n-1]} y_{i}=\max _{y \in[m]^{n-1}} \sum_{i \in[n-1]} y_{i}-h-1 .
$$

Expressions (3) and (4) are less elegant than Sander's expression (1). They are also computationally less efficient. Indeed the first summation in (3) has approximately $\mathrm{nm} / 2$ terms while the only summation in (1) has approximately $n / 2$ terms. Expressions (3) and (4) are nevertheless interesting because they allow us to derive an asymptotic result for $S(m, n)$ when $n$ is fixed and $m \rightarrow \infty$ (see Theorem 3). This was not possible with (1).

When $n<5$, expressions (3) and (4) reduce to particularly simple expressions. 


\section{Corollary 1}

$$
\begin{aligned}
& S(m, 2)=m ; \\
& S(m, 3)= \begin{cases}\frac{3 m^{2}}{4} & \text { if } m \text { is even, } \\
\frac{3 m^{2}+1}{4} \text { if } m \text { is odd }\end{cases} \\
& S(m, 4)=\frac{2 m^{3}+m}{3} .
\end{aligned}
$$

For $n=2,3$ or 4 , the asympotic behaviour of $S(m, n)$ is easy to derive from this corollary, while the general case is covered by our next result.

Theorem 3 For all $n \geq 2$, when $m \rightarrow \infty, S(m, n)$ is equal to $m^{n-1} \gamma(n)+$ $\mathcal{O}\left(m^{n-2}\right)$ where $\gamma(n)$ is equal to

$$
1-2 \sum_{j=0}^{\frac{n-4}{2}} \sum_{i=0}^{j}(-1)^{i} \frac{(1+j-i)^{n-1}-(j-i)^{n-1}}{i !(n-1-i) !}
$$

when $n$ is even, or to

$$
\begin{aligned}
& 1-2 \sum_{j=0}^{\frac{n-5}{2}} \sum_{i=0}^{j}(-1)^{i} \frac{(1+j-i)^{n-1}-(j-i)^{n-1}}{i !(n-1-i) !} \\
&-2 \sum_{i=0}^{\frac{n-3}{2}}(-1)^{i} \frac{\left(\frac{n}{2}-1-i\right)^{n-1}-\left(\frac{n-3}{2}-i\right)^{n-1}}{i !(n-1-i) !}
\end{aligned}
$$

when $n$ is odd.

Proof. We first prove (9). Expression (3) for $S(m, n)$ can also be written as

$$
\begin{array}{r}
m^{n-1}-2\left(\sum_{j=0}^{\frac{n-4}{2}} \sum_{r=n-1+j(m-1)}^{m+n-3+j(m-1)} \sum_{i=0}^{j}(-1)^{i}\left(\begin{array}{c}
n-1 \\
i
\end{array}\right)\left(\begin{array}{c}
r-i m-1 \\
r-i m-n+1
\end{array}\right)\right. \\
\left.+\sum_{r=n-1+\frac{n-2}{2}(m-1)} \sum_{i=0}^{\frac{n-2}{2}}(-1)^{i}\left(\begin{array}{c}
n-1 \\
i
\end{array}\right)\left(\begin{array}{c}
r-i m-1 \\
r-i m-n+1
\end{array}\right)\right)
\end{array}
$$

For $n$ fixed, $\gamma(n)$ is the limit for $n \rightarrow \infty$ of (11) divided by $m^{n-1}$, that is

$$
\begin{aligned}
& 1-\lim _{m \rightarrow \infty} \frac{2}{(n-2) ! m^{n-1}}\left(\sum_{j=0}^{\frac{n-4}{2}} \sum_{i=0}^{j}(-1)^{i}\left(\begin{array}{c}
n-1 \\
i
\end{array}\right) \sum_{r=n-1+j(m-1)}^{m+n-3+j(m-1)} \frac{(r-i m-1) !}{(r-i m-n+1) !}\right. \\
& \left.+\sum_{i=0}^{\frac{n-2}{2}}(-1)^{i}\left(\begin{array}{c}
n-1 \\
i
\end{array}\right) \sum_{r=n-1+\frac{n-2}{2}(m-1)}^{h-m-1} \frac{(r-i m-1) !}{(r-i m-n+1) !}\right) .
\end{aligned}
$$


Since $\lim _{a \rightarrow \infty} \frac{a ! /(a-b) !}{a^{b}}=1$, this is also equal to

$$
\begin{aligned}
& 1-\lim _{m \rightarrow \infty} \frac{2}{(n-2) ! m^{n-1}}\left(\sum_{j=0}^{\frac{n-4}{2}} \sum_{i=0}^{j}(-1)^{i}\left(\begin{array}{c}
n-1 \\
i
\end{array}\right) \sum_{r=n-1+j(m-1)}^{m+n-3+j(m-1)}(r-i m)^{n-2}\right. \\
& \left.+\sum_{i=0}^{\frac{n-2}{2}}(-1)^{i}\left(\begin{array}{c}
n-1 \\
i
\end{array}\right) \sum_{r=n-1+\frac{n-2}{2}(m-1)}^{h-m-1}(r-i m)^{n-2}\right)
\end{aligned}
$$

or, since $\sum_{d=a}^{b} d^{c}=(c+1)^{-1}\left[d^{c+1}\right]_{a}^{b}+\mathcal{O}\left(d^{c}\right)$ as $d \rightarrow \infty$,

$$
\begin{gathered}
1-\lim _{m \rightarrow \infty} \frac{2}{(n-1) ! m^{n-1}}\left(\sum_{j=0}^{\frac{n-4}{2}} \sum_{i=0}^{j}(-1)^{i}\left(\begin{array}{c}
n-1 \\
i
\end{array}\right)\left[(r-i m)^{n-1}\right]_{r=n-1+j(m-1)}^{m+n-3+j(m-1)}\right. \\
\left.+\sum_{i=0}^{\frac{n-4}{2}}(-1)^{i}\left(\begin{array}{c}
n-1 \\
i
\end{array}\right)\left[(r-i m)^{n-1}\right]_{r=n-1+\frac{(n-2)}{2}(m-1)}^{h-m-1}\right) .
\end{gathered}
$$

We then substitute $r$ with the summation bounds, we simplify and we take the limit, keeping only the coefficients of the highest power of $m$ (i.e. $m^{n-1}$ ) and we obtain (9). The second highest power of $m$ is $m^{n-2}$ and this completes the proof of $(9)$.

The reasoning for proving (10) is identical but starts from (4) instead of $(3)$.

Numerical estimations of $\gamma(n)$ are given in Table 2 and Figure 1 illustrates how quickly $S(m, n) / m^{n-1}$ converges to $\gamma(n)$ for $n=10$ and 100. Expressions (9) and (10) are easy to compute for $n$ between 2 and 100. Our implementation of (9) in Python takes approximately 0.029 seconds to compute $\gamma(100)$. For $100<n<1000$, computing $\gamma(n)$ with our implementation of $\gamma(n)$ takes longer but is still achievable (see Table 2). Computation of $\gamma(n)$ for $n$ larger than 1000 becomes problematic.

\begin{tabular}{rlrrlr}
\hline$n$ & $\gamma(n)$ & time & $n$ & $\gamma(n)$ & time \\
\hline 2 & 1 & 0.000 & 9 & 0.45292096819196426 & 0.000 \\
3 & $3 / 4$ & 0.000 & 10 & 0.43041776895943573 & 0.000 \\
4 & $2 / 3$ & 0.000 & 50 & 0.19485381202305646 & 0.004 \\
5 & $115 / 192$ & 0.000 & 100 & 0.13799020407550003 & 0.029 \\
6 & $11 / 20$ & 0.000 & 500 & 0.06178532829673273 & 8.984 \\
7 & 0.5110243055555556 & 0.000 & 1000 & 0.04369538142621430 & 174.987 \\
8 & 0.47936507936507944 & 0.000 & 1500 & 0.03567891389083611 & 1123.630 \\
\hline
\end{tabular}

Table 2 Some values of $\gamma(n)$. Fractions are exact. Computing time in seconds.

Notice that, when $n<5$, Corollary 1 provides an asymptotic expression for $S(m, n)$ that is tighter than that resulting from Theorem 3 . 

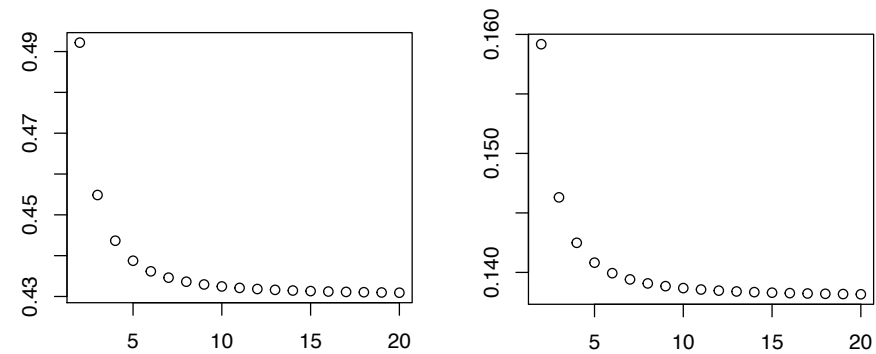

Fig. 1 Hor. axis: $m$ (from 2 to 20 ). Vert. axis: $S(m, n) / m^{n-1}$. Left: $n=10$; right: $n=100$.

\section{Conflict of interest}

The authors declare that they have no conflict of interest.

\section{References}

1. Bouyssou, D., Marchant, T., Pirlot, M.: A theoretical look at ELECTRE TRI-nB (2020). URL https://hal .archives-ouvertes.fr/hal-02898131. Working paper hal-02898131

2. Branzei, R., Llorca, N., Sánchez-Soriano, J., Tijs, S.: A constrained egalitarian solution for convex multi-choice games. TOP 22, 860-874 (2014)

3. De Bruijn, N.G., Van Ebbenhorst Tengbergen, C., Kruyswijk, D.: On the set of divisors of a number. Nieuw Archief voor Wiskunde 23, 191-193 (1951)

4. Ersek Uyanık, E., Sobrie, O., Mousseau, V., Pirlot, M.: Enumerating and categorizing positive Boolean functions separable by a $k$-additive capacity. Discrete Applied Mathematics 229, 17-30 (2017)

5. Fernández, E., Figueira, J.R., Navarro, J., Roy, B.: ELECTRE TRI-nB: A new multiple criteria ordinal classification method. European Journal of Operational Research 263(1), 214-224 (2017)

6. Freixas, J.: The Banzhaf value for cooperative and simple multichoice games. Group Decision and Negotiation 29, 61-74 (2020)

7. Grabisch, M.: Remarkable polyhedra related to set functions, games and capacities. TOP 24(2), 301-326 (2016)

8. Griggs, J.R.: Maximum antichains in the product of chains. Order 1, 21-28 (1984)

9. Hsiao, C.R., Liao, Y.H.: The potential and consistency property for multi-choice Shapley value. Taiwanese Journal of Mathematics 12(2), 545-559 (2008)

10. Hsiao, C.R., Raghavan, T.E.S.: Monotonicity and dummy free property for multi-choice cooperative games. International Journal of Game Theory 21, 301-312 (1992)

11. Kim, H.K.: On regular polytope numbers. Proceedings of the American Mathematical Society 131(1), 65-75 (2002)

12. Mattner, L., Roos, B.: Maximal probabilities of convolution powers of discrete uniform distributions. Statistics and Probability Letters 78, 2992-2996 (2008)

13. de Moivre, A.: The doctrine of chances. Third ed., reprinted by Chelsea, New York, 1967 (1756)

14. Motek, J.: Problem 86-8. The Mathematical Intelligencer 8 (1986)

15. OEIS: The On-line Encyclopaedia of Integer Sequences, Sloane, N. J. A. (Ed.) (2019). URL https://oeis.org

16. Proctor, R.A., Saks, M.E., Sturtevant, D.G.: Product partial orders with the Sperner property. Discrete Mathematics 30, 173-180 (1980)

17. Sander, J.W.: On maximal antihierarchic sets of integers. Discrete Mathematics 113, 179-189 (1993) 
18. Scott, B.M.: Width of a product of chains, https://math.stackexchange.com/ questions/299770 (2013). Last Checked: March 5, 2020

19. Sperner, E.: Ein Satz über Untermengen einer endlichen Menge. Mathematische Zeitschrift 27(1), 544-548 (1928)

20. Tsai, S.F.: A simple upper bound on the number of antichains in $[t]^{n}$. Order (2018). DOI 10.1007/s11083-018-9480-5. URL https://doi.org/10.1007/s11083-018-9480-5 